\title{
Experimental research of a four-stroke piston type compressed air engine
}

\author{
Fancong Zeng ${ }^{1}$, Jinli $\mathrm{Xu}^{1}$, and Rui Zhao ${ }^{1}$ \\ ${ }^{1}$ School of Mechanical and Electrical Engineering, Wuhan University of Technology, Wuhan, China
}

\begin{abstract}
The purpose of this paper is to investigate working performance for a four-stroke piston type compressed air engine (CAE) via experimental research. A conventional internal combustion engine(IC engine) is modified to a four-stroke CAE and a mathematical model is set up. Working performances are investigated by output torque, power, air consumption, exhaust pressure, exhaust temperature and cylinder pressure on a test bench. The CAE is installed on a vehicle for the road test. The experiment and analysis show several meaningful results. The increase of the supply pressure will result in the increase of the rotational speed, and it can effectively improve the output torque and power. However, it can lead to an increase in air consumption. The success of this application demonstrates compressed air cars are likely to be industrialized duo to their zero pollution and zero emissions.
\end{abstract}

\section{Introduction}

Over the recent decades, environmental protection and reducing carbon dioxide $\left(\mathrm{CO}_{2}\right)$ emission become crucial issues worldwide. Exhaust gas from internal combustion (IC) engines contributes largely to $\mathrm{CO}_{2}$ emissions, and IC engines pollute the environment seriously. In order to improve fuel consumption and protect the environment, researchers have been searching for environmentally friendly energies to replace conventional fossil fuels [1, 2]. Compressed air, as the working medium of the compressed air engine (CAE), is inexhaustible. A CAE has both the advantages of non-burning fuel and zero pollution, and there is no chemical reaction and high temperature in its working cycle [3, 4]. Compressed air can be generated through several ways, such as solar energy, wind energy and tidal energy [5], which does not rely on non-renewable energy.

Lots of literatures on the CAE have been investigated. Zhang et al. [6] studied the valve timing for optimizing the efficiency and performance of the CAE. An optimized polynomial cam profile was applied and the influences of the half wrap angle and the maximum valve lift to the fullness coefficient were studied. Song et al. [7] established the mathematic model and dimensionless of the $\mathrm{CAE}$, and the influences of the non-dimensional cylinder stroke and Kagawa coefficient on CAE's performances were analyzed. The research results showed that the non-dimensional cylinder stroke had little effect on the CAE, but the heat transfer has a remarkable effect on the performance of the CAE. The faster the heat transfer was, the larger the efficiency and power were. Huang et al. [8] presented an experimental investigation of a CAE. The CAE was a modified $100 \mathrm{~cm}^{3}$ two-stroke IC engine obtained from a motorcycle manufacturer. The experiments in this study used a test bench to examine the power performance and pressure/temperature variations of the CAE at pressures ranging from 5 to 9 bar.

In this paper, an experimental research of a fourstroke piston type compressed air engine was carried out, the influences on the output torque, power, air consumption, exhaust pressure, exhaust temperature and cylinder pressure were analyzed with the variations in rotational speed and supply pressure. Therefore, the experimental research of the four-stroke CAE is carried out in this paper for performance analysis and the design of the CAE.

\section{Mathematical model of the CAE}

As shown in Figure. 1, inlet valves and exhaust valves are controlled by the valve system. At the beginning of the intake stroke, the intake valves open immediately, and the atmosphere flows into the cylinder through the inlet valves because of the pressure difference. When the piston is near the bottom dead center (BDC), the inlet valves close. In the compression stroke, the piston moves up near the top dead center (TDC). In the inflation and expansion stroke, the intake valve and the air nozzle begin to work, then the compressed air flows into the cylinder through the air nozzle, and pushes the piston downwards. After a specific crank angle, the intake valve and the air nozzle close while the compressed air expands to push the piston down and output work. When the piston moves back to the bottom dead center (BDC), the exhaust valves open so that residual air discharges under the impetus of the piston. After the piston moves back to the top dead center (TDC), the four-stroke CAE completes a complete work cycle. 
The CAE cylinder can be seen to be a thermodynamic system, and the following assumptions are made. First, the compressed air is ideal, which means specific heat and specific enthalpy are only related to the temperature. Second, the changes of kinetic and gravitational energy are negligible. Third, the process of gas flowing into/out of the cylinder is a quasi-steady one. Finally, there is no leak or friction during the working process.

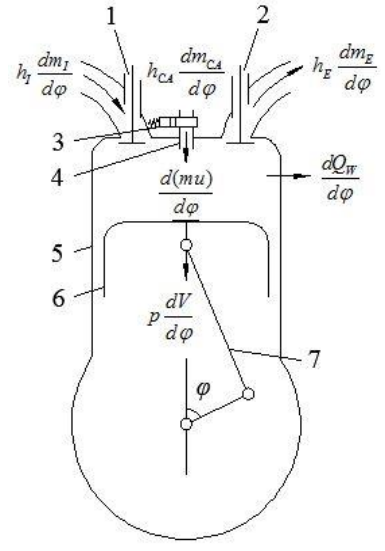

1. Inlet valve 2. Exhaust valve 3. Intake valve 4. Air nozzle 5. Cylinder 6. Piston 7. Crank-link mechanism

Fig. 1. Structure and thermodynamic analysis diagram of fourstroke CAE

The energy equation can be expressed as

$$
\frac{d U}{d \varphi}=\frac{d Q_{w}}{d \varphi}+h_{I} \frac{d m_{I}}{d \varphi}+h_{C A} \frac{d m_{C A}}{d \varphi}+h_{E} \frac{d m_{E}}{d \varphi}+\frac{d W}{d \varphi}
$$

Where $U$ is the internal energy of the air in the cylinder; $Q_{w}$ is the heat absorbed by the air from the cylinder walls; $h_{j}(j=I, E, C A)$ are the specific enthalpies of inlet air, exhaust air and injection compressed air, respectively; $m_{j}(j=I, E, C A)$ are the mass of inlet air, exhaust air and injection compressed air, respectively; $W$ is the mechanical work on the piston; $\varphi$ is the crank angle.

$U$ can be written in this form

$$
d U=d(m \cdot u)=u d m+m d u
$$

Where $m$ is the mass of the air in the cylinder; $u$ is the specific heat.

For ideal air, $u$ can be seen as a function of air temperature, that is $u=u(T)$, and $u$ can be written in a fully differential form

$$
\frac{d u}{d \varphi}=\frac{\partial u}{\partial T} \frac{d T}{d \varphi}=c_{v} \frac{d T}{d \varphi}
$$

Where $c_{v}$ is the constant volume specific heat; $T$ is the temperature of the air in the cylinder.

The heat transfer in the process can be expressed as

$$
\frac{d Q_{w}}{d \varphi}=\frac{1}{\omega} \sum_{i=1}^{3} a_{g} \cdot A_{i}\left(T_{w i}-T\right)
$$

Where $\omega$ is the angular speed of the crank shaft; $a_{g}$ is the heat transfer coefficient; $A_{i}$ is the total heat transfer area; $T_{w i}(i=1,2,3)$ are the temperatures of the cylinder head, the piston and the cylinder liner, respectively.

The coefficient of the heat transfer between the air and the cylinder walls is given as

$$
a_{g}=0.1129 \cdot D^{-0.2} \cdot p^{0.8} \cdot(S n / 30)^{0.8} \cdot T^{-0.594}
$$

Where $D$ is the diameter of the cylinder; $p$ is the pressure of the air in the cylinder; $S$ is the stroke of the piston; $n$ is the rotational speed of the CAE.

The mechanical work by the compressed air is described by

$$
\frac{d W}{d \varphi}=-p \frac{d V}{d \varphi}
$$

Where $V$ is the cylinder volume, $V$ can be written as

$$
\begin{aligned}
V= & \frac{\pi D^{2}}{4}\left\{\frac{S}{\varepsilon-1}+\frac{S}{2}\left[\left(1+\frac{1}{\lambda}\right)\right.\right. \\
& \left.\left.-\cos \varphi-\frac{1}{\lambda} \sqrt{1-\lambda^{2} \cdot \sin ^{2} \varphi}\right]\right\}
\end{aligned}
$$

Where $\varepsilon$ is the compression ratio; $\lambda$ is the ratio of crank radius to connecting rod.

The change rate of the cylinder volume can be expressed as

$$
\frac{d V}{d \varphi}=\frac{\pi \cdot D^{2} \cdot S}{8}\left[\sin \varphi+\frac{\lambda}{2} \frac{\sin (2 \cdot \varphi)}{\sqrt{1-\lambda^{2} \sin ^{2} \varphi}}\right]
$$

Substituting equations (2)-(6) into equation (1) yields the following differential equation

$$
\begin{aligned}
\frac{d T}{d \varphi} & =\frac{1}{m \cdot c_{v}}\left(\frac{d Q_{w}}{d \varphi}-p \frac{d V}{d \varphi}+h_{I} \cdot \frac{d m_{I}}{d \varphi}\right. \\
& \left.+h_{C A} \cdot \frac{d m_{C A}}{d \varphi}+h_{E} \cdot \frac{d m_{E}}{d \varphi}-u \frac{d m}{d \varphi}\right)
\end{aligned}
$$

The mass air flow of the CAE can be expressed as

$$
\frac{d m_{j}}{d \varphi}=\frac{1}{\omega} \cdot \mu F \sqrt{\frac{p_{1}}{v_{1}}} \cdot \psi
$$

Where $\mu$ is the coefficient of discharge; $F$ is the effective sectional area; $p_{1}$ is the upstream pressure; $v_{1}$ is the upstream air specific volume; $\psi$ is the flow function.

When $\frac{p_{r}}{p_{1}}>\left(\frac{2}{k+1}\right)^{\frac{k}{k-1}}$, the air flow state is sub-sonic flow state, and the flow function $\psi$ can be written as

$$
\psi=\sqrt{\frac{2 k}{k-1}\left[\left(\frac{p_{r}}{p_{1}}\right)^{\frac{2}{k}}-\left(\frac{p_{r}}{p_{1}}\right)^{\frac{k+1}{k}}\right]}
$$

Where $k$ is the adiabatic exponent of the air; $p_{r}$ is the downstream pressure. 
When $\frac{p_{r}}{p_{1}} \leq\left(\frac{2}{k+1}\right)^{\frac{k}{k-1}}$, the air flow state is sonic flow state, and the flow function $\psi$ can be written as

$$
\psi=\left(\frac{2}{k+1}\right)^{\frac{1}{k-1}} \sqrt{\frac{2 k}{k+1}}
$$

The ideal gas equation of state can be derived as

$$
p V=m R T
$$

Here, $R$ is the gas constant of air.

\section{Experimental method}

In this study, the type of the CAE is a four-stroke piston type CAE, which is modified from a four-stroke IC engine. It can output the mechanical energy transformed from the compressed air energy into the torque form. The four-stroke CAE system is mainly composed of main air supply system, branch air supply system, electronic control system and CAE body. Figure 2 is the physical map of the four-stroke CAE system. The main air supply system is connected with air supply pipes through its main flow meter, which is combined with the branch air supply system. The main air supply system stores highpressure compressed air prepared by an air compressor in air tanks, which acts as the power medium of the CAE. The branch air supply system fills the compressed air into four cylinders, respectively, and the compressed air is obtained through the high-pressure and large-flow pressure reducing valve and controlled by four intake valves. The electronic control system is divided into two parts: ECU electronic control system and bench test system. ECU electronic control system mainly refers to the control system of ECU development platform of the CAE, and also involves the control of timing and pulse width of the intake valves, and can realize the data acquisition and real-time display of the air pressure and air flow of the CAE. The platform test system can collect and measure the cylinder pressure, the exhaust pressure and the exhaust temperature when the CAE is working, and the output torque and power can also be measured.

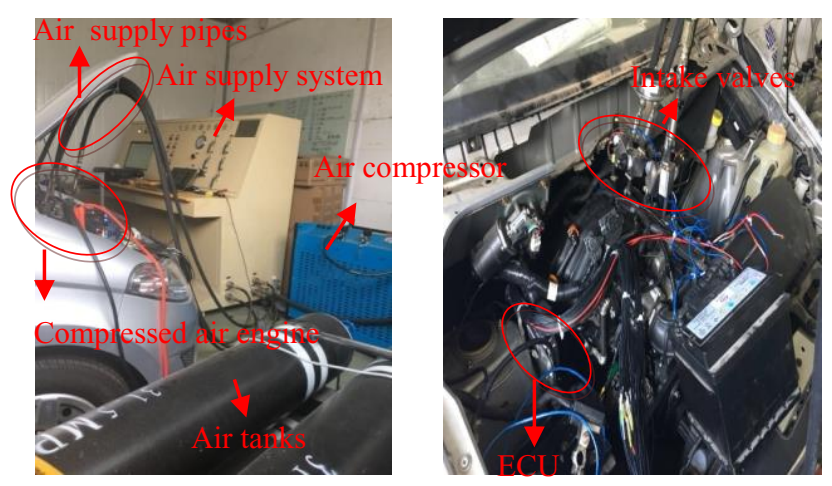

Fig. 2. Physical map of the four-stroke CAE system.

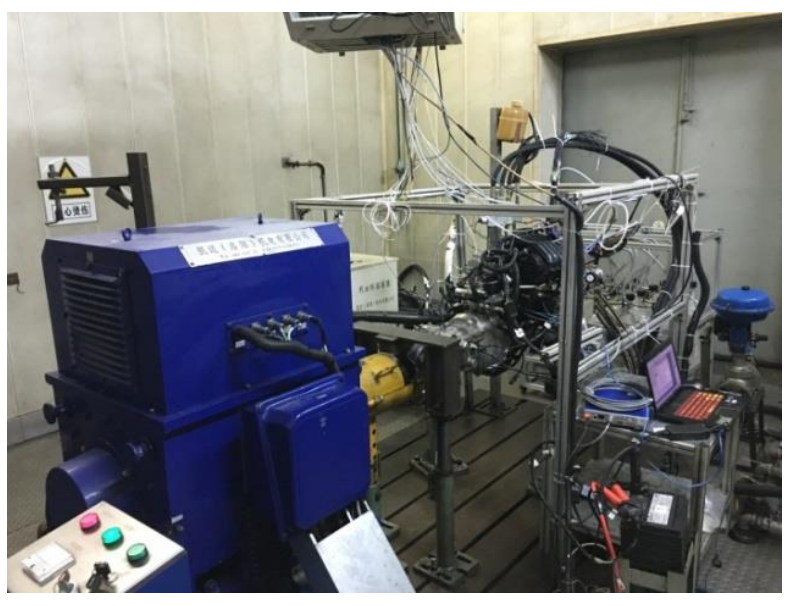

Fig. 3. CAE test bench.

In order to verify the performance of the four-stroke $\mathrm{CAE}$, calibration software and data acquisition system are used to test the CAE on the test bench, the CAE test bench is shown in figure 3 and the measurement and control system is shown in figure 4 . The debugged four-stroke CAE is installed on the vehicle for the road test to test the operation of the compressed air car under different working conditions. Figure 5 shows the compressed air car developed in this study. The following conclusions can be obtained through the road test: (1) Vibration and noise of the CAE are much less than that of traditional IC engine, and the operation stability is well. (2) The prototype car can run easily when it is in first gear, the supply pressure required in the initial stage is bigger than that in the process of driving. At the same time, driving speed can be significantly increased by increasing the supply pressure and reducing the weight of the car. (3) Since the prototype car is a rear wheel drive car and it is very heavy, the CAE will shake after shifting gears, the rotational speed can't reach higher speed range.

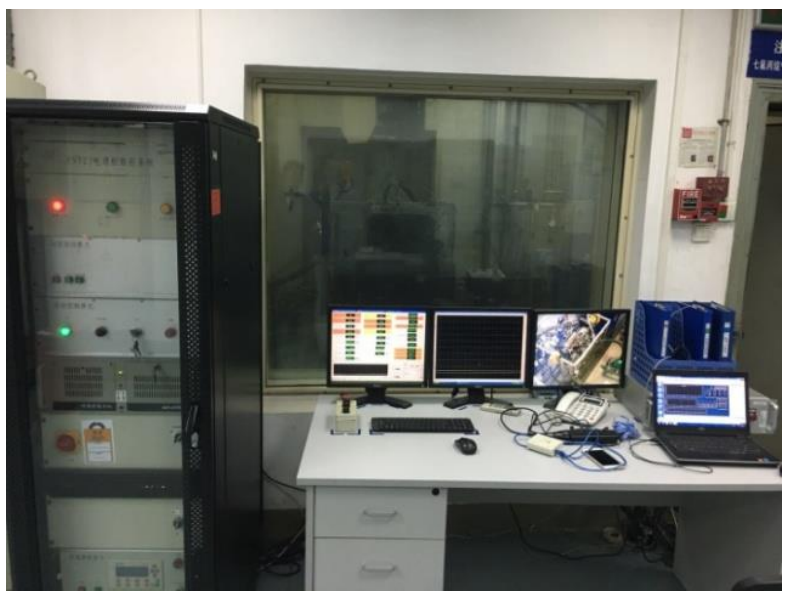

Fig. 4. Measurement and control system. 


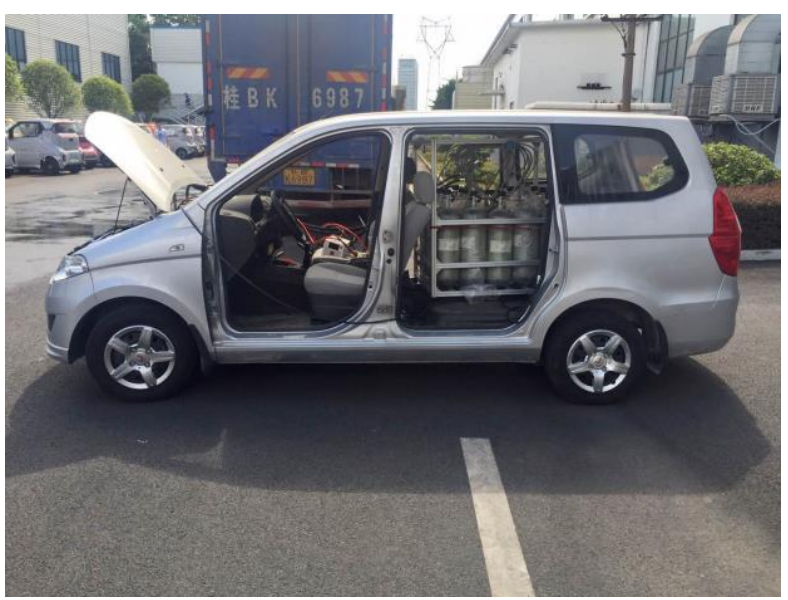

Fig. 5. Compressed air car.

\section{Result and discussion}

Working characteristics of the four-stroke CAE can be investigated by output torque, power, air consumption, exhaust pressure, exhaust temperature and cylinder pressure. Taking the rotational speed as the independent variable, the variation of each important performance parameter with the rotational speed is discussed in this paper. The supply pressure is set to 20bar and 30bar, respectively, and the resistance torque is set to $2 \mathrm{Nm}$, the performance parameters are recorded at different rotational speeds and the test results are shown in figure $6 \sim$ figure 9. From these figures, it can be found that the higher the rotational speed, the smaller the output torque but the larger the power and air consumption. When running at a lower rotational speed, it consumes less air and has better economic performance. The exhaust pressure and the exhaust temperature gradually increase with the rising of rotational speed for the same supply pressure. With the increasing of output torque, the exhaust temperature will become lower, even below zero. The larger the output torque, the faster the exhaust temperature decreases. The increase of supply pressure will result in the increase of output torque, power and air consumption and the decrease of exhaust temperature.

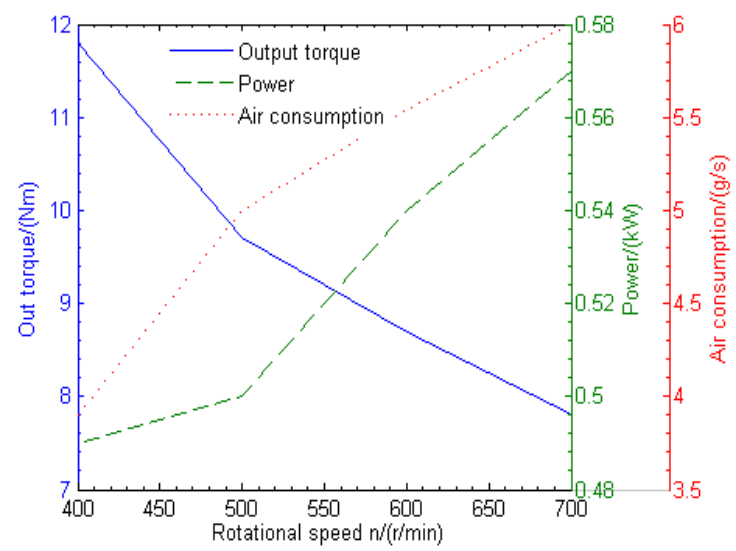

Fig. 6. Variations of output torque, power and air consumption at different rotational speeds. (20bar)

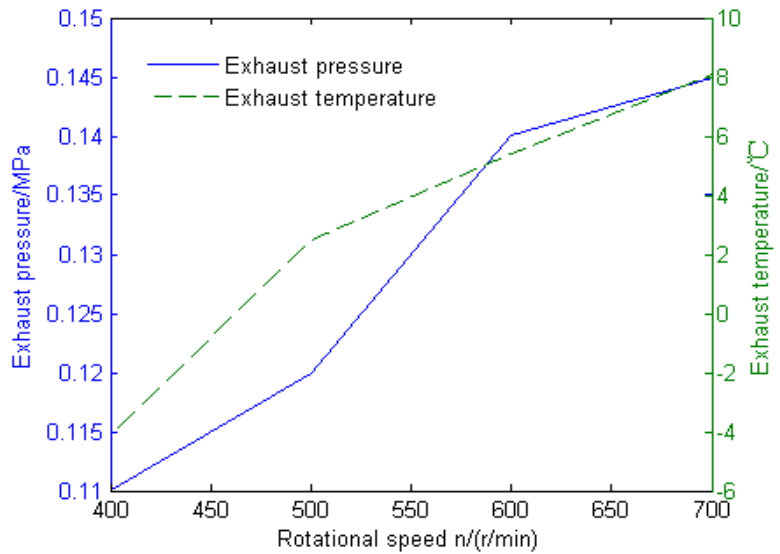

Fig. 7. Variations of exhaust pressure and exhaust temperature at different rotational speeds. (20bar)

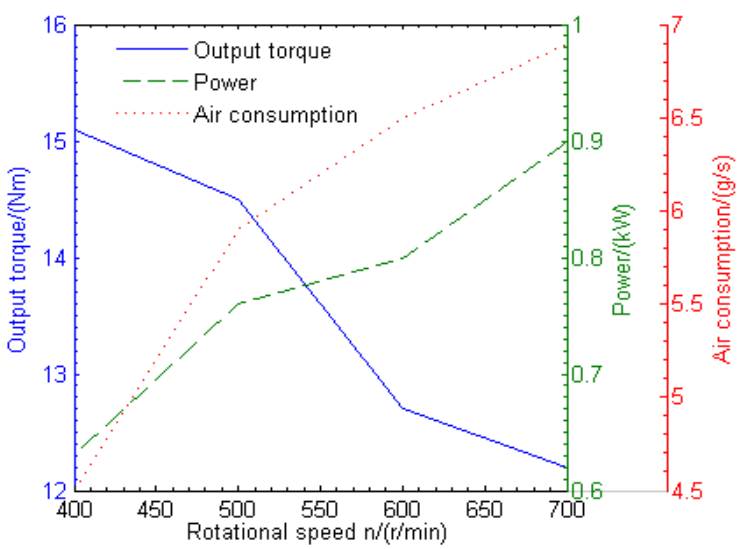

Fig. 8. Variations of output torque, power and air consumption at different rotational speeds. (30bar)

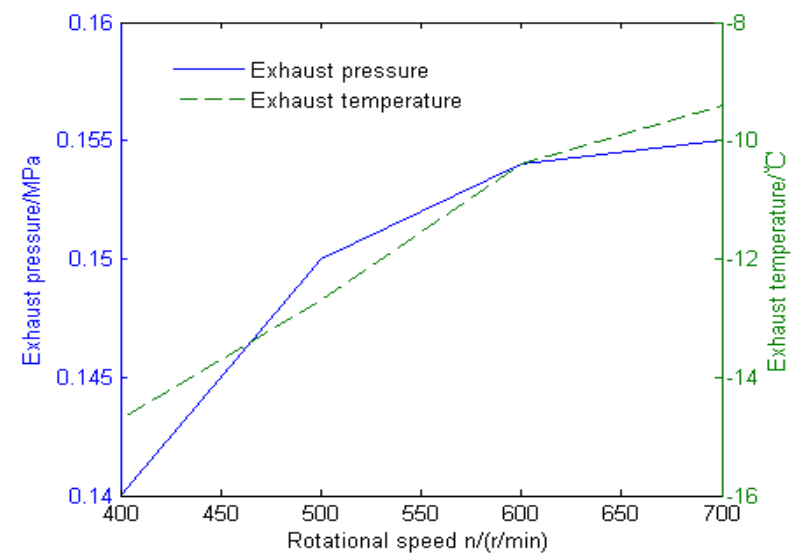

Fig. 9. Variations of exhaust pressure and exhaust temperature at different rotational speeds. (30bar)

As shown in figure 10, the supply pressure is set to $20 \mathrm{bar}$ and 30bar, respectively, and the variations of cylinder pressure in a working cycle are revealed. The cylinder pressure increases rapidly in the compression stage $\left(180^{\circ} \mathrm{CA} \sim 355^{\circ} \mathrm{CA}\right)$, and the peak cylinder pressures are about $16.5 \mathrm{bar}$ and $25 \mathrm{bar}$, respectively, the cylinder pressure drops rapidly in the expansion stage $\left(405^{\circ} \mathrm{CA} \sim 530^{\circ} \mathrm{CA}\right)$. 


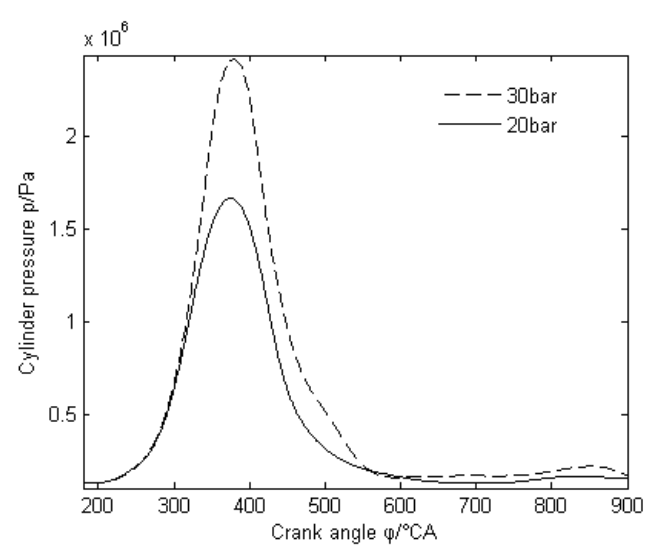

Fig. 10. Cylinder pressure plot.

The peak cylinder pressure is lower than the supply pressure, mainly due to the following reasons:

(1) Although the supply pressure is certain during the test, the air flow rate is smaller than that of the theory due to the limitation of the air flow rate of the intake valves.

(2) The compressed air diffuses gradually in the cylinder, and the cylinder pressure sensors are installed far away from the center of the air-filled flow field, the measured cylinder pressure will be lower than the peak cylinder pressure.

(3) Due to the delays in the opening and closing of the intake valves, and there will be air throttling in the pipeline from the outlets of the intake valves to the jets of the CAE. The piston is already in the downward process when the air is filled into the cylinder, and the cylinder volume gradually increases. At this point, the flow of insufficient compressed air into the cylinder will reduce the cylinder pressure.

(4) Although relevant seal processing has been done in the modification of the CAE, there is still gap between piston ring and cylinder diameter surface. The mounting holes of the valves and cylinder pressure sensors are not completely sealed either, these are the channels of air leakage, which directly lead to insufficient cylinder pressure.

In conclusion, when the air flow is larger, the time of establishing cylinder pressure will be shorter and the peak pressure will be greater. Therefore, the improvement and optimization of the four-stroke CAE in cylinder and valve will be the focus of future research.

Driving torque and resistance torque of the CAE are balanced during stable operation. By comparing and analyzing the variation of the rotational speed with the supply pressure when the CAE is under no load and $2 \mathrm{Nm}$ load, the characteristic of the resistance torque is investigated, thus reflecting the power performance. The variations of rotational speed at different supply pressures are shown in figure 11. As observed from this figure, the rotational speed at no load is obviously higher than that at $2 \mathrm{Nm}$ load under a certain supply pressure, and the higher the supply pressure, the greater the difference between the rotational speeds of no load and $2 \mathrm{Nm}$ load. This indicates that the $\mathrm{CAE}$ needs to consume more pressure energy when overcoming the load to output mechanical work, and the rotational speed and power can be improved by increasing the supply pressure and air flow rate of compressed air.

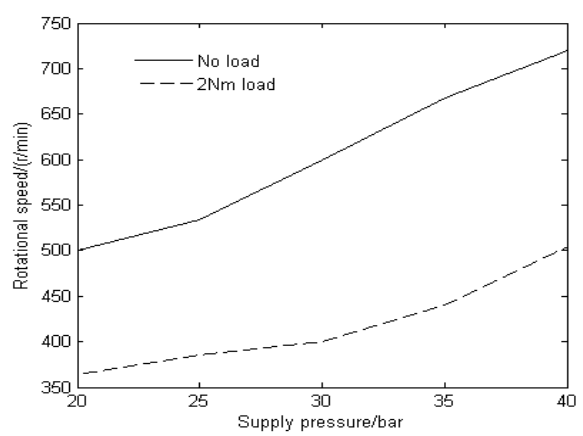

Fig. 11. Variation of rotational speed at different supply pressures.

\section{Conclusions}

In this paper, a four-stroke piston type CAE is used to investigate the working performance. An experimental system is developed to research the influence of several key performance parameters on working characteristics, and the success of car test drive with four-stroke CAE demonstrates the feasibility of vehicle applications. The CAE has higher output torque, lower power and air consumption in lower rotational speed stage, and the exhaust pressure and the exhaust temperature gradually increase with the rising of rotational speed. The increase of the supply pressure can effectively improve power performance. However, compressed air car has limited range and power, due to the low energy density of compressed air. Therefore, there are still many problems to be solved in the application of the CAE as main power system on vehicles, and the study of this work will provide a certain guidance function for further study on optimizing design of the CAE.

The authors would like to thank the automobile company SGMW in China for its kind assistance with the four-stroke IC engine fused for the present study.

\section{References}

1. X.L. Yuan, X. Liu, J. Zuo, Renew. Sust. Energ. Rev 42, 8 (2015)

2. F.C. Zeng, J.L. Xu, Therm. Sci 22, 13 (2018)

3. D. Marvaniaa, S. Subudhi, Renew. Sust. Energ. Rev 70, 12 (2017)

4. A. Papson, F. Creutzig, L. Schipper, Transport. Res. Rec 93, 8 (2010)

5. Z.G. Xu, X.P. Xie, IEEE, 5 (2009)

6. Z. Zhang, R.B. Jia, Q.H. Yu, M.L. Cai, AMM, 278280, 5 (2013)

7. R.Z. Song, X. H. Fu, M.L. Cai, AMM, 278-280, 8 (2013)

8. C.Y. Huang, C.K. Hu, C.J. Yu, C.K. Sung, Energies 6, 15 (2013) 